\title{
Phtalates: new cardiovascular health disruptors?
}

\author{
Giovanna Muscogiuri $^{1} \cdot$ Annamaria Colao $^{2}$
}

Received: 6 May 2016 / Accepted: 21 June 2016 / Published online: 29 June 2016

(C) Springer-Verlag Berlin Heidelberg 2016

\begin{abstract}
Phtalates are commonly found in several household products such as food packaging, furniture and toys. Humans are exposed to phtalates through different ways such as inhalation, ingestion and dermal contact. Due to the abundance of plastic in our society, the exposure to phtalates is ubiquitous. A growing body of evidence investigated the association of phtalate exposure with cardiovascular risk factors, i.e., obesity, type 2 diabetes and hypertension. Phtalates are thought to contribute to obesity through their binding and activation of PPAR $\gamma$ receptor that in turn results in the upregulation of adipocyte production. Phtalates are also known to interfere with insulin signaling and to increase oxidative stress. All these mechanisms contribute to the onset of insulin resistance. Recent evidences support a role of phtalates in the pathogenesis of atherosclerosis and hypertension. Thus, the aim of this communication was to summarize the current evidences dealing with the association of phtalates and cardiovascular risk factors.
\end{abstract}

Keywords Phtalates Environment $\cdot$ Lifestyle $\cdot$ Type 2 diabetes $\cdot$ Cardiovascular disease $\cdot$ Obesity

Phtalates can be found in a variety of products such as cosmetics, shampoos, soaps, lubricants, pesticides and paints. Food is the main tool responsible for the human exposure

Giovanna Muscogiuri

giovanna.muscogiuri@gmail.com

1 Ios and Coleman Medicina Futura Medical Center, Via Alcide De Gasperi, 107, 80011 Acerra (NA), Italy

2 Department of Clinical Medicine and Surgery (A.C.), University "Federico II", via Sergio Pansini, 5, 80131 Naples, Italy to phthalates that are often contained in wrapping materials or during the food processing (Wams 1987). The metabolic transformation of phtalates after the exposure starts with hydrolytic breakup of diesters (Meeker et al. 2012). The hydrolytic monoesters that result from this molecular step are then excreted in the urine or are further processed as glucuronide conjugates, some of which are oxidized. The main metabolites of phtalates are: di-2-ethylhexyl phthalate (DEHP), mono-ethylphthalate (MEP), monobutyl phthalate (MBP), mono-benzylphthalate (MBzP), mono-(2-ethylhexyl) phthalate (MEHP), mono-(2-ethyl5-hydroxyhexyl) phthalate (MEHHP), mono-(2-ethyl-5-oxohexyl) phthalate (MEOHP), mono-(2-ethyl-5-carboxypentyl) phthalate (MECPP), mono-isobutyl phthalate (MiBP), mono-(3-carboxypropyl) phthalate(MCPP) (Frederiksen et al. 2007). The free monoesters, glucuronide conjugates and the oxidative metabolites are used as urinary markers of phtalate exposure (Kato et al. 2004). A growing body of studies focused on the phtalate-related diseases. A link has been found between phtalate exposure and cancer (Erkekoglu and Kocer-Gumusel 2014), developmental outcomes (Bay et al. 2006), thyroid function (Andra and Makris 2012) and fertility (Meeker 2010). Recent evidences pointed out phtalates as cardiovascular health disruptors. This hypothesis was based on studies that reported an association of phtalate exposure with cardiovascular risk factors such as obesity, lipid metabolism, blood pressure and atherosclerosis.

The correlation between phtalates and obesity has been assessed in several studies. Most of the results come from the NHANES studies and surveys carried out in the USA in order to obtain a random sample of the civilian noninstitutionalized population for a given survey cycle. Stalhult et al. (2007) by measuring waist circumference (WC) in adult the US male enrolled in the National Health and 
Nutrition Examination Survey (NHANES) 1999-2002 investigated whether the phthalate metabolites MBP, MBzP, MEHP, MEP, MEHHP and MEOHP were predictor of abdominal obesity. MBzP, MEHHP, MEOHP and MEP have been identified as predictor of abdominal obesity, thus suggesting that the exposure to these phtalate metabolites may contribute to the burden of obesity (Stahlhut et al. 2007). This study included only male subjects while Hatch et al. subsequently analyzed the same NHANES database but including both genders (Hatch et al. 2008). The endpoints of interest in Hatch's study were BMI and WC.

The main findings were the association of MBzP, MEOHP, MEHHP, MEP and MBP with BMI and WC in males aged 20-59 and the association of MEP to BMI and WC in adolescent girls (Hatch et al. 2008). Trasande et al. (2013a, b) focused their study on the 2003-2008 National Health and Nutrition Examination Survey performing a cross-sectional analysis of 2884 children (aged 6-19 years). The endpoints of interest were 2000 CDC reference-based BMI z-scores and binary variables of overweight and obesity that were defined using BMI z-score cutoffs of 85th and 95th percentile, respectively. The interesting finding of this study was that the association between anthropometric measures with phtalate metabolites was found to be race/ethnicity specific. In fact each $\log$ unit increase in low molecular weight metabolites was associated with 21 and $22 \%$ increases in odds (95\% CI $1.05-1.39$ and 1.07-1.39, respectively) of overweight and obesity and a 0.090 -SD unit increase in BMI z-score (95\% CI 0.003-0.18), among non-Hispanic blacks but not in other racial/ethnic subgroup (Trasande et al. 2013a, b). Using data from the Prospective Investigation of the Vasculature in Uppsala Seniors (PIVUS) study, Lind et al. assessed whether circulating concentrations of phthalates were related to different indices of obesity (Lind et al. 2012a). Serum phtalate metabolites levels were assessed at baseline and dual-energy X-ray absorptiometry (DXA), and abdominal magnetic resonance imaging (MRI) assessments were performed 2 years later. The results of Lind et al. were presented separately by gender. In women, MiBP levels were positively correlated with WC, total fat mass and trunk fat mass assessed by DXA, as well as to subcutaneous adipose tissue assessed by MRI while monomethyl phthalate (MMP) concentrations were related to trunk fat mass and the trunk/leg-ratio assessed by DXA, although this association was less powerful than the association with MiBP. No association between phtalate metabolites and anthropometric indices was found in men. The interesting study performed by Lind et al. has the limit that could not be considered as a truly longitudinal study and this is because exposure assessment was performed 2 years before the outcome ascertainment; thus, the follow-up status of participants could not be corresponding to the status at baseline (Lind et al. 2012a) (Fig. 1).

The association of phtalates and phtalate metabolites as indices of obesity could be explained by a molecular viewpoint. In fact, MEHP directly binds and activates PPAR $\gamma$, thus promoting adipogenesis. Importantly, MEHP induces a selective activation of different PPAR $\gamma$ co-regulators including Med1 and PGC-1 $\alpha$, but not p300 and SRC (Feige et al. 2007). In addition to PPAR $\gamma$, phthalate monoesters have been reported to activate PPAR $\alpha$ and PPAR $\beta$ both in mice and humans with similar sensitivity (Bility et al. 2004). Hurst et al. investigated the activation of PPAR $\alpha$ and $\operatorname{PPAR} \gamma$ by phthalate monoesters using cell-based transactivation assays and by monitoring PPAR target gene expression (PPAR $\alpha$ ) or PPAR-dependent adipocyte differentiation (PPAR $\gamma$ ) (Hurst and Waxman 2003). Among the phtalate monoesters, MBzP and MEHP have been shown to activate both human and mouse PPAR $\alpha$ and PPAR $\gamma$ while mono-methyl, mono- $n$-butyl, dimethyl or diethyl esters of phthalic acid did not (Hurst and Waxman 2003). Although the exposure of 3T3-L1 cell model to MEHP induced expression of PPAR $\gamma$ as well as its target genes, Hao et al. aimed to demonstrate whether this results were confirmed in vivo and in particular whether perinatal exposure to MEHP may have any effect on filial adipogenesis (Hao et al. 2012). At postnatal day 60, an increased in adipose tissue deposition was observed in male offspring of mother exposed to MEHP during pregnancy, thus showing that in vivo results were consistent with in vitro results (Hao et al. 2012).

Phtalates and their metabolites also seem to be involved in glucose derangements. A cross-sectional study performed by Svensson et al. (2011) assessed the association between phtalate and self-reported diabetes in 221 healthy Mexican women that were previously enrolled as controls in an on-going case-control study for breast cancer. Higher concentrations of DEHP metabolites such as MEHHP, MEOHP and MECPP but lower levels of MBzP have been found in patients with diabetes compared to participants without diabetes (Svensson et al. 2011). These results also were confirmed by James-Todd et al. (2012) in a crosssectional study on a large population 2350 women between 20 and 79 years of age who participated in the NHANES (2001-2008). In this study, the endpoint of interest also was self-reported diabetes. After dividing the population in quartiles, women with higher levels of mono-n-butyl phthalate (MnBP),MiBP, MBzP, MCPP and three di-(2- ethylhexyl) phthalate metabolites ( $\Sigma$ DEHP) had an increased odds of diabetes compared with women with the lowest levels of these phthalates. Women in the highest quartile for MBzP and MiBP had almost twice the odds of diabetes compared with women in the lowest quartile (JamesTodd et al. 2012). Lind et al. (2012b) reported an increased 
prevalence of diabetes with increasing urinary excretion of MMP, MiBP and MEP in 1016 subjects, aged 70 years, belonging to the Prospective Investigation of the Vasculature in Uppsala Seniors (PIVUS) Study. In particular, MiBP was mainly related to decreased insulin secretion assessed by fasting proinsulin-to-insulin ratio, whereas MEP and MMP mainly were related to insulin resistance assessed by HOMA index (Lind et al. 2012b). In the same cohort of study, Olsén et al. (Olsén et al. 2012) found a significant association between MiBP and fasting glucose. There are few studies that provide an explanation of the link between glucose derangements and exposure to phtalates and their metabolites from a molecular viewpoint. As well known, an imbalance between the oxidative stress production and antioxidant defenses may play an important role in inducing alterations in insulin signaling pathways (Lamb and Goldstein 2008). Marker of oxidative stress such as malondialdehyde has been reported to be increased by exposure to phthalates and to correlate in a dose-response manner with $\sum$ DEHP and insulin resistance index (Kim et al. 2013). The hypothesis that could explain this link is that exposure to phthalates induces mitochondrial dysfunction and thus oxidative stress leading to the onset of insulin resistance. Latter, phtalates have been reported to decrease serum insulin levels in rats administered with DEHP at a low level of $750 \mathrm{microg} / 100 \mathrm{~g}$ body weight on alternate days for 14 days (Gayathri et al. 2004) and to interfere with insulin signaling in adipose tissue of Wistar rats (Rajesh et al. 2013). All these pathological mechanisms could lead to rises in blood glucose (Fig. 1).

Phtalates and their metabolites have been reported to be associated with blood pressure and hypertension. An accurate assessment of PIVUS study revealed a significant inverse correlation between MEP and both systolic (SBP) and diastolic blood pressure (DBP) (Olsén et al. 2012). Phtalate metabolites such as MBP, MEHHP, MEOHP, MEHP and LDEHP were associated with SBP, but none of these metabolites was related to DBP in the Trasande et al. study (Trasande et al. 2013b).

A tight relation between DEHP and blood pressure was found in a subsample of US children aged 6-19 years enrolled in the National Health and Nutrition Examination Survey between 2003 and 2008. For each log unit increase in DEHP metabolites, a 0.041 SD unit increase in systolic BP z-score was identified (Trasande et al. 2013b). MBzP concentrations have been associated with increased DBP

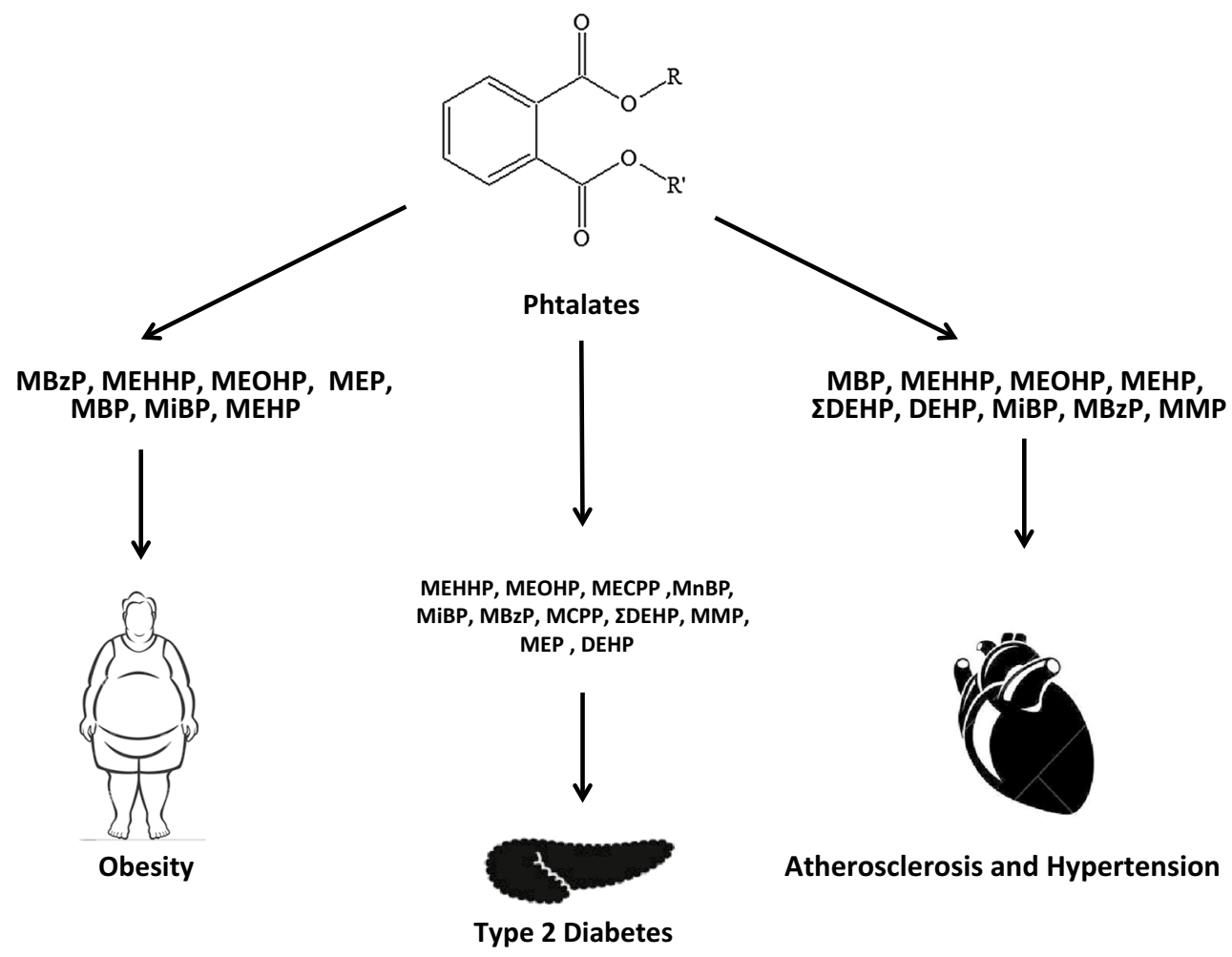

Fig. 1 Summary of phtalate metabolites associated with obesity, type 2 diabetes, atherosclerosis and hypertension. Abbr. di-2-ethylhexyl phthalate (DEHP), mono-ethylphthalate (MEP), mono-butyl phthalate (MBP), mono-benzylphthalate (MBzP), mono-(2-ethylhexyl) phthalate (MEHP), mono-(2-ethyl-5-hydroxyhexyl) phthalate (MEHHP), mono-(2-ethyl-5-oxohexyl) phthalate (MEOHP), mono-(2-ethyl5-carboxypentyl) phthalate (MECPP), mono-isobutyl phthalate (MiBP), mono-(3-carboxypropyl) phthalate(MCPP), mono-n-butyl phthalate (MnBP), mono-methyl phthalate (MMP), di-(2- ethylhexyl) phthalate metabolites ( $\Sigma$ DEHP) 
and risk of pregnancy-induced hypertensive diseases in 369 women of the Health Outcomes and Measures of the Environment Study, a prospective birth cohort of low-risk pregnant women recruited between March 2003 and January 2006 (Werner et al. 2015). The phtalate-related hypertension could be related to the increased plaque echogenicity and intima-media thickening and echogenicity that are more likely to happen in subjects exposed to phtalates (Lind and Lind 2011). In the study performed by Lind et al. (2011), MMP was related to the development of carotid plaques in an inverted U-shaped manner while MiBP and MMP levels were associated with the echogenicity of the plaques.

The current evidence on the association between phtalates and cardiovascular risk factors is of little-high quality. This is due to the fact that phtalates have short physiologic half-lives; thus, the single measurement performed in most of the studies cannot provide information on long-term exposure. Further, since phtalates have a large number of metabolites and the investigators that conducted studies on phtalates measured not the effects of the same metabolites, it is hard to get a comparison of the results across the studies. Moreover, the majority of the studies are based upon population-based surveys or pharmacovigilance studies, i.e., studies that were not designed to address the effect of phtalates on cardiovascular risk factors.

In summary, we conclude that the design of the studies and exposure assessment limitations make hard to get definitive conclusions on the effect of phtalates on cardiovascular health. Due to the short half-lives of phtalates, longitudinal studies assessing the relation between repeated measures of phtalate exposure and repeated health followup may provide insightful evidence about the effect of phtalates exposure on cardiovascular health.

\section{References}

Andra SS, Makris KC (2012) Thyroid disrupting chemicals in plastic additives and thyroid health. J Environ Sci Health C Environ Carcinog Ecotoxicol Rev. 30(2):107-151

Bay K, Asklund C, Skakkebaek NE, Andersson AM (2006) Testicular dysgenesis syndrome: possible role of endocrine disrupters. Best Pract Res Clin Endocrinol Metab 20(1):77-90

Bility MT, Thompson JT, McKee RH, David RM, Butala JH, Vanden Heuvel JP, Peters JM (2004) Activation of mouse and human peroxisome proliferator-activated receptors (PPARs) by phthalate monoesters. Toxicol Sci 82(1):170-182

Erkekoglu P, Kocer-Gumusel B (2014) Genotoxicity of phthalates. Toxicol Mech Methods 24(9):616-626

Feige JN, Gelman L, Rossi D, Zoete V, Métivier R, Tudor C, Anghel SI, Grosdidier A, Lathion C, Engelborghs Y, Michielin O, Wahli W, Desvergne B (2007) The endocrine disruptor monoethylhexyl-phthalate is a selective peroxisome proliferator-activated receptor gamma modulator that promotes adipogenesis. J Biol Chem 282(26):19152-19166
Frederiksen H, Skakkebaek NE, Andersson AM (2007) Metabolism of phthalates in humans. Mol Nutr Food Res 51(7):899-911

Gayathri NS, Dhanya CR, Indu AR, Kurup PA (2004) Changes in some hormones by low doses of di (2-ethyl hexyl) phthalate (DEHP), a commonly used plasticizer in PVC blood storage bags and medical tubing. Indian J Med Res 119(4):139-144

Hao C, Cheng X, Xia H, Ma X (2012) The endocrine disruptor mono(2-ethylhexyl) phthalate promotes adipocyte differentiation and induces obesity in mice. Biosci Rep 32(6):619-629

Hatch EE, Nelson JW, Qureshi MM, Weinberg J, Moore LL, Singer M, Webster TF (2008) Association of urinary phthalate metabolite concentrations with body mass index and waist circumference: a cross-sectional study of NHANES data, 1999-2002. Environ Heal 7:27

Hurst CH, Waxman DJ (2003) Activation of PPARalpha and PPARgamma by environmental phthalate monoesters. Toxicol Sci 74(2):297-308

James-Todd T, Stahlhut R, Meeker JD, Powell SG, Hauser R, Huang T, Rich-Edwards J (2012) Urinary phthalate metabolite concentrations and diabetes among women in the National Health and Nutrition Examination Survey (NHANES) 2001-2008. Environ Heal Perspect 120(9):1307-1313

Kato K, Silva MJ, Reidy JA, Hurtz D 3rd, Malek NA, Needham LL, Nakazawa H, Barr DB, Calafat AM (2004) Mono(2-ethyl-5-hydroxyhexyl) phthalate and mono-(2-ethyl-5-oxohexyl) phthalate as biomarkers for human exposure assessment to di-(2-ethylhexyl) phthalate. Environ Heal Perspect 112(3):327-330

Kim JH, Park HY, Bae S, Lim YH, Hong YC. (2013) Diethylhexyl phthalates is associated with insulin resistance via oxidative stress in the elderly: a panel study. PLoS One. 19;8(8):e71392

Lamb RE, Goldstein BJ (2008) Modulating an oxidative-inflammatory cascade: potential new treatment strategy for improving glucose metabolism, insulin resistance, and vascular function. Int $\mathrm{J}$ Clin Pract 62(7): 1087-1095

Lind PM, Lind L (2011) Circulating levels of bisphenol A and phthalates are related to carotid atherosclerosis in the elderly. Atherosclerosis. 218(1):207-213

Lind PM, Roos V, Rönn M, Johansson L, Ahlström H, Kullberg J, Lind L (2012a) Serum concentrations of phthalate metabolites are related to abdominal fat distribution two years later in elderly women. Environ Heal 11:21

Lind PM, Zethelius B, Lind L (2012b) Circulating levels of phthalate metabolites are associated with prevalent diabetes in the elderly. Diabetes Care 35(7):1519-1524

Meeker JD (2010) Exposure to environmental endocrine disrupting compounds and men's health. Maturitas 66(3):236-241

Meeker JD, Calafat AM, Hauser R (2012) Urinary phthalate metabolites and their biotransformation products: predictors and temporal variability among men and women. J Expo Sci Environ Epidemiol. 22(4):376-385

Olsén L, Lind L, Lind PM (2012) Associations between circulating levels of bisphenol $\mathrm{A}$ and phthalate metabolites and coronary risk in the elderly. Ecotoxicol Environ Saf 80:179-183

Rajesh P, Sathish S, Srinivasan C, Selvaraj J, Balasubramanian K (2013) Phthalate is associated with insulin resistance in adipose tissue of male rat: role of antioxidant vitamins. J Cell Biochem 114(3):558-569

Stahlhut RW, van Wijngaarden E, Dye TD, Cook S, Swan SH (2007) Concentrations of urinary phthalate metabolites are associated with increased waist circumference and insulin resistance in adult U.S. males. Environ Heal Perspect 115(6):876-882

Svensson K, Hernández-Ramírez RU, Burguete-García A, Cebrián ME, Calafat AM, Needham LL, Claudio L, López-Carrillo L (2011) Phthalate exposure associated with self-reported diabetes among Mexican women. Environ Res 111(6):792-796 
Trasande L, Attina TM, Sathyanarayana S, Spanier AJ, Blustein J (2013a) Race/ethnicity-specific associations of urinary phthalates with childhood body mass in a nationally representative sample. Environ Heal Perspect 121(4):501-506

Trasande L, Sathyanarayana S, Spanier AJ, Trachtman H, Attina TM, Urbina EM (2013b) Urinary phthalates are associated with higher blood pressure in childhood. J Pediatr 163(3):747-753
Wams TJ (1987) Diethylhexylphthalate as an environmental contaminant-a review. Sci Total Environ 66:1-16

Werner EF, Braun JM, Yolton K, Khoury JC, Lanphear BP (2015) The association between maternal urinary phthalate concentrations and blood pressure in pregnancy: the HOME Study. Environ Heal 14:75 